\title{
Severe transient neutropenia with fever and abdominal pain in a 30-year-old man
}

\author{
Sarah Hudgins MD, Alexander Wong MD
}

$\mathrm{A}$ 30 -year-old man presented to the emergency department with a 3-day history of fever, chills, nausea, vomiting, diarrhea and abdominal pain. The patient's symptoms had progressed rapidly until he had a productive cough with yellow sputum, which led him to visit the emergency department.

The patient's medical history included hepatitis C, asthma and cholecystectomy. Results of serologic testing for HIV 4 months earlier had been negative. The patient was taking ipratropium and salbutamol. In addition, he stated that he regularly used both cocaine and morphine intravenously.

On physical examination, the patient appeared unwell. Although he was drowsy, he was easily arousable. His temperature was $38.7^{\circ} \mathrm{C}$, with a blood pressure of 70/44 $\mathrm{mm} \mathrm{Hg}$ and a pulse of 93 beats/min. He did not have any rashes, and his oropharynx was unremarkable. He had no cervical, axillary or inguinal lymphadenopathy. His chest examination was unremarkable, except for a mild inspiratory wheeze. The patient's heart sounds were normal, with no additional sounds or murmurs. His abdomen was diffusely tender to palpation, but there was no rebound tenderness or guarding, nor was there evidence of hepatosplenomegaly or palpable masses. His right knee showed signs of recent trauma.

The results of initial laboratory investigations showed a leukocyte count of 0.7 (normal 4.0 $11.0) \times 10^{9} / \mu \mathrm{L}$ and a neutrophil count of 0.4 (normal 2.0-7.5) $\times 10^{\%} / \mu \mathrm{L}$. Liver enzyme testing showed an alanine aminotransferase concentration of 48 (normal 4-55) U/L and an aspartate aminotransferase concentration of 179 (normal 5-35) U/L. We admitted the patient to hospital, where he received piperacillin-tazobactam for febrile neutropenia; however, his neutropenia spontaneously remitted the following day. His condition responded quickly to aggressive supportive measures, including fluids delivered intravenously. After 1 day, the patient's leukocyte count had risen to $20.7 \times 10^{9} / \mu \mathrm{L}$, and his neutrophil count was $19.1 \times 10^{9} / \mu \mathrm{L}$. Cultures of blood drawn before antibiotics were administered grew Bacillus cereus in 1 of 2 vials after 16 hours, prompting us to add vancomycin to the drug regimen, pending susceptibility testing. Chest radiography showed patchy airspace disease in the lower lobe of the patient's left lung. A computed tomography scan of the abdomen and pelvis showed a normal liver and biliary tree, diverticulosis without diverticulitis, a trace right pleural effusion and a trace amount of free pelvic fluid.

\section{What is your differential diagnosis?}

a. Mononucleosis secondary to Epstein-Barr virus (EBV) or cytomegalovirus (CMV)

b. Acute hepatitis A infection

c. Acute HIV infection

d. Levamisole toxicity secondary to adulterated cocaine

e. Endocarditis secondary to B. cereus

We thought that an infectious agent was the most likely explanation for our patient's unusual symptoms, specifically (c) HIV or (d) B. cereus. Given the unusual presentation of his condition, however, we ordered serologic tests for EBV and $\mathrm{CMV}$, which were negative. Although the results of serologic tests for these 2 viruses can be negative early in the course of disease, we did not perform serial testing once an alternative diagnosis had been made.

The results of serologic testing for HIV were indeterminate, but the results of a Western blot test were negative. Hence, we arranged further testing to rule out the possibility of acute HIV infection.

Laboratory investigations showed no immunoglobulin $\mathrm{M}$ against the hepatitis $\mathrm{A}$ virus, but the presence of immunoglobulin $\mathrm{G}$ against the hepatitis A virus suggested pre-existing immunity. Repeat blood cultures showed no growth, and a transesophageal echocardiogram showed no evidence of valvular vegetation.
Competing interests: None declared.

This article has been peer reviewed

Correspondence to:

Alexander Wong, alexander.wong@usask.ca

CMAJ 2013. DOI:10.1503 /cmaj.111951 
Levamisole-induced granulocytopenia secondary to the use of adulterated cocaine (d) has been well reported and usually causes bone marrow suppression that lasts about 7 days, often necessitating the use of granulocyte colonystimulating factor. ${ }^{1,2}$ We thought that the rapid and spontaneous resolution of our patient's neutropenia was unlikely to be compatible with levamisole toxicity.

The patient's fever resolved after several days. On the 5th day of his stay in hospital, the antibiotic agents he was taking were changed to levofloxacin to treat community-acquired pneumonia, given his cough and the results of his chest radiograph. His initial positive blood culture was considered to be the result of contamination.

Within 2 days after starting levofloxacin, the patient again began to experience high fevers $\left(>40^{\circ} \mathrm{C}\right.$ ) and worsening abdominal pain, in addition to substantial mucocutaneous ulcerations in his oropharynx. We stopped treatment with levofloxacin and restarted piperacillin-tazobactam. Although the patient's liver enzymes were only moderately elevated (alanine aminotransferase $62 \mathrm{U} / \mathrm{L}$, aspartate aminotransferase $92 \mathrm{U} / \mathrm{L}$ ), his cholestatic markers had risen substantially since admission (alkaline phosphatase 454 [normal 40-135] U/L, $\gamma$-glutamyltransferase 1111 [normal 0-51] IU/L). We consulted with the gastroenterology service for endoscopic retrograde cholangiopancreatography, the results of which were normal. However, the patient's esophagus was markedly abnormal (Figure 1).

\section{What do these findings suggest?}

a. CMV esophagitis

b. Candida esophagitis

c. Herpes simplex virus esophagitis

d. Medication-induced esophagitis

e. Eosinophilic esophagitis

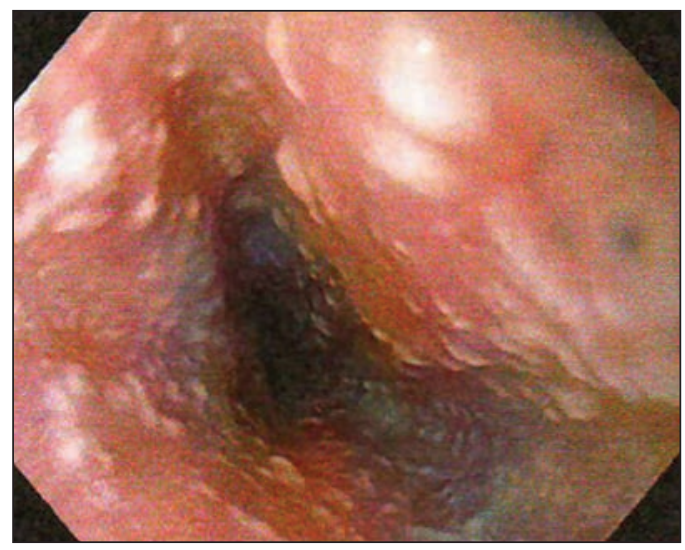

Figure 1: Midesophageal lesions in 30-year-old man with transient febrile neutropenia, nausea, vomiting, diarrhea, elevated liver enzymes and abdominal pain.
The presence of diffuse, raised, white, plaquelike lesions was considered pathognomonic for esophageal candidiasis (b), and we started treatment with fluconazole immediately. Pathologic examination of esophageal biopsies taken during endoscopy confirmed this diagnosis.

Esophagitis secondary to infection with herpes simplex virus or CMV typically presents as small mucosal ulcerations on endoscopy. ${ }^{3}$ Endoscopic findings of eosinophilic esophagitis include a narrowed esophageal lumen and mucosal rings with white papules. ${ }^{4}$ None of these entities present with raised whitish plaques, as we saw in our patient. Medication-induced esophagitis is rare and most often associated with the use of alendronate, tetracycline antibiotics and antiinflammatory medications, none of which our patient had been exposed to recently. ${ }^{3}$

The presence of esophageal candidiasis further raised our concern about acute HIV infection. Results of tests for the p24 antigen using the sera gathered for initial HIV testing were positive, confirming the diagnosis of acute HIV seroconversion. Antibiotic therapy was stopped, and repeat HIV serologic testing by Western blot showed positive results with a viral load of 2342485 copies/mL. We considered starting antiretroviral medications immediately, but this course of action was deferred at the patient's request.

\section{Discussion}

Neutropenia is defined as an absolute neutrophil count that is 2 standard deviations below the normal population mean. In teenagers and adults, this amounts to an absolute neutrophil count of less than $1.8 \times 10^{3} / \mu \mathrm{L}$. An absolute neutrophil count of less than 500 cells $/ \mu \mathrm{L}$ is considered severe and increases a patient's susceptibility to infections. ${ }^{5,6}$ "Transient" neutropenia distinguishes between neutropenia that resolves in a short time (usually without intervention other than elimination of the underlying cause) and neutropenia of a more prolonged duration.

Depending on the cause of neutropenia and the adequacy of the patient's bone marrow reserve, the risk of infection may increase as the absolute neutrophil count decreases. A patient with adequate bone marrow reserve and the ability to deliver neutrophils to the site of infection may be at no increased risk of infection, despite having limited neutrophils in the peripheral blood. ${ }^{6}$ In high-risk scenarios where bone marrow reserves are clearly decreased or absent, such as with chemotherapy-induced neutropenia or bone marrow infiltration, a patient with fever and neutropenia is at risk of overwhelming sep- 
sis and should receive treatment immediately with broad-spectrum antibiotics to cover both gram-positive and gram-negative organisms. Evidence-based guidelines have recently been updated to guide clinical decision-making in this patient population.?

The most common causes of nonmalignancyrelated neutropenia are listed in Box 1.5,6 Bone marrow reserves may be normal or decreased, according to the underlying cause, which then determines a patient's risk of infection. The results of a physical examination can provide important clues regarding the adequacy of a patient's bone marrow reserve. The presence of gingivitis or mucosal ulcerations strongly suggests inadequate bone marrow reserve, although these findings can be seen independently in various forms of vasculitis, as well as in primary HIV infection. The presence of any purulent material in a patient with neutropenia strongly suggests adequate bone marrow reserve. However, if a patient presents with fever and neutropenia of unknown cause with equivocal or absent signs on physical examination, clinicians must assume that the patient is at high risk for infection and treat the condition appropriately with broad-spectrum antibiotics.

Recent guidelines outline the initial clinical investigations to be done when patients present with febrile neutropenia. ${ }^{7}$ In brief, routine laboratory investigations should be performed, including a complete blood count with differential, serum creatinine and blood urea nitrogen, electrolytes, and hepatic enzymes with total bilirubin. The initial results of these investigations can help to determine the presence of multiorgan dysfunction and the appropriate dosing of medications, including empiric antibiotics. A minimum of 2 blood cultures is recommended, with simultaneous samples for culture drawn from a central venous catheter (if present) and a peripheral vein site. If a central venous catheter is not present, 2 blood samples from different peripheral vein sites should be drawn. A chest radiograph should be taken for patients with respiratory symptoms, and samples from other sites can be obtained for culture as clinically indicated. If the cause of neutropenia is unclear, further diagnostics can be obtained based on the differential diagnoses listed in Box 1.

\section{Acute HIV Infection}

Acute HIV infection is associated with high plasma viremia, which declines rapidly upon antibody seroconversion. Most patients with acute HIV infection experience symptoms, but these are nonspecific and may be attributed to some other "viral illness" by both patients and clinicians. Patients with symptoms of HIV infection will nearly all have fever, and many will have lymphadenopathy that develops shortly after their initial symptoms. Painful mucocutaneous disease is a hallmark sign and should raise clinical suspicion for acute HIV infection. Gastrointestinal symptoms are common, as is headache. Opportunistic infections traditionally attributed to advanced immunosuppression in late-stage HIV such as Pneumocystis jirovicii pneumonia and esophageal candidiasis can be seen during acute HIV infection, as with our patient, although this is less common. ${ }^{8,9}$ The presence of any such opportunistic infection in the absence of a clear reason for immunosuppression warrants consideration for HIV testing. ${ }^{10}$

Standard enzyme-linked immunoassays to detect the presence of anti-HIV antibodies are the most commonly used screening tests, with high sensitivity (> 99.5\%) but a substantial rate of false-positive results. ${ }^{11}$ Positive screens are followed by a confirmatory Western blot analysis with high specificity (>99.5\%), which excludes false-positive tests. ${ }^{11} \mathrm{~A}$ diagnostic "window period" after which infection has occurred but before antibodies have reached detectable levels makes it difficult to diagnose acute HIV infection using standard screening assays. Advances in diagnostic testing have allowed a reduction in this period to an average of 22 days with current third-generation antibody assays. ${ }^{11}$ Fourthgeneration assays that combine testing for the p24 antigen and anti-HIV antibodies further reduce this window to an average of 16 days.. ${ }^{11}$

\section{Box 1: Nonmalignant causes of neutropenia ${ }^{5,6}$}

Inadequate bone marrow reserve and disorders of neutrophil production

- Immunosuppressing agents, such as methotrexate and cyclosporine (most common cause of neutropenia in the United States) $)^{5}$

- Myelodysplastic syndrome, aplastic anemia

- Congenital disorders (Kostmann syndrome, glycogen storage diseases, cyclic neutropenia, congenital immunodeficiencies, Chédiak-Higashi syndrome)

- Vitamin $B_{12}$ or folic acid deficiency

- Chronic idiopathic adult neutropenia

Adequate bone marrow reserve and disorders of neutrophil use and turnover

- Alloimmune neonatal neutropenia

- Autoimmune disorders (systemic lupus erythematosus, rheumatoid arthritis, Felty syndrome, Sjogren syndrome)

- Drug-induced secondary to clozapine, antithyroid drugs, indomethacin, penicillins, clindamycin, gentamicin, phenytoin, amitriptyline, chloroquine, hydrochlorothiazide, levamisole (rare; drug-induced neutropenia occurs in 3-12 cases per 1 million population per year ${ }^{5}$ )

- Infection (e.g., hepatitis B virus, hepatitis C virus, Epstein-Barr virus, HIV parasites, tuberculosis, malaria)

- Hypersplenism 
In light of the many possible clinical manifestations of acute primary HIV infection and the practical difficulties of identifying patients at high risk, it is important for clinicians to maintain a low threshold for testing in patients with a compatible presentation. If there is clinical suspicion of acute HIV seroconversion in a high-risk patient and standard antibody testing is either negative or indeterminate, then further virologic testing (either p24 antigen capture or an HIV RNA assay) should be pursued. ${ }^{12}$ HIV RNA is usually detectable by polymerase chain reaction 2 weeks after infection. However, many laboratories will not perform an HIV RNA assay after negative or indeterminate antibody test results, because the assay is expensive and both labourand time-intensive. Clinicians are advised to contact their laboratories to discuss individual scenarios on a case-by-case basis. If a patient receives a diagnosis of acute HIV infection while their antibody status remains negative or indeterminate, repeat serology should be performed over the next 3 months to confirm seroconversion. Early treatment of HIV is now recommended and should be offered to all patients with acute infection. ${ }^{13}$ Consultation with a medical microbiologist or infectious diseases specialist may be beneficial.

Very few cases of severe transient neutropenia associated with acute HIV infection appear in the literature, and all of them reported simultaneous thrombocytopenia and rapid spontaneous resolution of neutropenia. ${ }^{14-18}$ In each of these cases, the patients received a short course of empiric antimicrobial therapy, as did our patient. We did not perform a biopsy of our patient's bone marrow, but in cases where bone marrow aspiration was performed, the results have essentially been normal. ${ }^{15-17}$ This would suggest a peripheral mechanism of neutropenia, possibly secondary to the presence of either antineutrophil or antigranulocyte antibodies.

\section{Conclusion}

Our patient's case highlights practical challenges in both the management of transient febrile neutropenia and the diagnosis of acute HIV infection. Clinicians should be aware of the dangers of severe neutropenia with fever and start treatment with broad-spectrum antibiotics after appropriate investigations have been performed in patients at high risk of infection and in those with an unclear cause for their neutropenia. Acute HIV infection usually presents in a nonspecific manner, and it is important to maintain high clinical suspicion to make the diagnosis. There are challenges in diagnosing acute HIV infection in light of the diagnostic window, and patients at high risk should undergo testing with either a p24 antigen capture assay or an HIV RNA assay in the absence of reactive serology. If clinical suspicion remains high despite initial negative testing, physicians should repeat appropriate serologic tests.

\section{References}

1. Zhu NY, Legatt DF, Turner AR. Agranulocytosis after consumption of cocaine adulterated with levamisole. Ann Intern Med 2009; 150:287-9.

2. Agranulocytosis associated with cocaine use — four states, March 2008-November 2009. MMWR Morb Mortal Wkly Rep 2009;58: 1381-5.

3. Katzka DA. Esophageal disorders caused by medications, trauma, and infection. In: Feldman M, Friedman LS, Brandt LJ, editors. Sleisenger and Fordtran's gastrointestinal and liver disease: pathophysiology, diagnosis, management. 9th ed. Philadelphia (PA): Saunders Elsevier; 2010;735-43.

4. Enns R, Kazemi P, Chung W, et al. Eosinophilic esophagitis: clinical features, endoscopic findings and response to treatment. Can J Gastroenterol 2010;24:547-51.

5. Dale DC. Neutropenia and neutrophilia. In: Prchal JT, Kaushansky K, Lichtman MA, et al., editors. Williams hematology. 8th ed. New York (NY): McGraw Hill; 2010. Available: www .accessmedicine.com/content.aspx ?aID=6133632 (accessed 2013 Mar. 6).

6. Dinauer MC, Coates TD. Disorders of phagocyte function and number. In: Hoffman R, editor. Hematology: basic principles and practice. 5th ed. Philadelphia (PA): Churchill Livingstone Elsevier; 2009;701-19.

7. Freifeld AG, Bow EJ, Sepkowitz KA, et al. Clinical practice guideline for the use of antimicrobial agents in neutropenic patients with cancer: 2010 update by the Infectious Diseases Society of America. Clin Infect Dis 2011;52:e56-93.

8. Cilla G, Perez Trallero E, Furundarena JR, et al. Esophageal candidiasis and immunodeficiency associated with acute HIV infection. AIDS 1988;2:399-400.

9. Vento S, Di Perri G, Garofano T, et al. Pneumocystis carinii pneumonia during primary HIV-1 infection. Lancet 1993;342:24-5.

10. Apoola A, Ahmad S, Radcliffe K. Primary HIV infection. Int $J$ STD AIDS 2002;13:71-8.

11. Dewar R, Goldstein D, Maldarelli F. Diagnosis of human immunodeficiency virus infection. In: Mandell GL, Bennett JE, Dolin R, editors. Mandell, Douglas and Bennett's principles and practice of infectious diseases. 7 th ed. Philadelphia (PA): Churchill Livingstone Elsevier; 2010;1663-86.

12. Stekler J, Maenza J, Stevens CE, et al. Screening for acute HIV infection: lessons learned. Clin Infect Dis 2007;44:459-61.

13. Panel on Antiretroviral Guidelines for Adults and Adolescents. Guidelines for the use of antiretroviral agents in HIV-1 infected adults and adolescents. Washington (DC): Departments of Health and Human Services; 2013. Available: www.aidsinfo.nih .gov/ContentFiles/AdultandAdolescentGL.pdf (accessed 2013 Mar. 23).

14. Colson P, Foucault C, Mokhtari M, et al. Severe transient neutropenia associated with acute human immunodeficiency virus type 1 infection. Eur J Intern Med 2005;16:120-2.

15. Ribera E, Ocaña I, Almirante B, et al. Autoimmune neutropenia and thrombocytopenia associated with development of antibodies to human immunodeficiency virus. J Infect 1989;18:167-70.

16. Skiest DJ, King ME. Granulocytopenia secondary to acute infection with the human immunodeficiency virus. J Infect 1994; 28:315-8.

17. Rousseau MC, Benesech M, Harlé JR. HIV seroconversion revealed by agranulocytosis. Presse Med 1998;27:161-2.

18. Ben-Galim P, Shaked Y, Vonsover A, et al. Immediate immunosuppression caused by acute HIV 1 infection: a fulminant multisystemic disease 2 days post-infection. Infection 1996;24: $332-5$.

Affiliations: Department of Obstetrics and Gynecology (Hudgins), Division of Infectious Diseases (Wong), University of Saskatchewan, Regina, Sask.

Contributors: Sarah Hudgins prepared the initial draft of the manuscript. Alexander Wong revised the manuscript for important intellectual content. Both authors approved the final version submitted for publication. 